\title{
Monitoring of Kidney Functions, Electrolytes and Volemia Analyzed by Application Example of Artificial Intelligence in Patients with Heart Failure
}

Danijela Tasić ( $\sim$ danijeladt@gmail.com )

University of Nis, Serbia, Medical Faculty, UCC Nis

Katarina Đorđević

University of Belgrade, Vinca Institute of Nuclear Sciences -National Institute of the Republic of Serbia

Slobodanka Galović

University of Belgrade, Vinca Institute of Nuclear Sciences -National Institute of the Republic of Serbia

Draško Furudžić

Institute "Mihajlo Pupin" Belgrade

Zorica Dimitrijević

University of Nis, Serbia, Medical Faculty, UCC Nis

Sonja Radenković

University of Nis, Serbia, Medical Faculty, UCC Nis

\section{Research Article}

Keywords: kidney, heart, electrolytes, congestion

Posted Date: December 28th, 2021

DOI: https://doi.org/10.21203/rs.3.rs-1162298/v1

License: (1) This work is licensed under a Creative Commons Attribution 4.0 International License.

Read Full License 


\section{Abstract}

Basal renal function is a predictor of response to diuretic therapy and marker of poor prognosis. Simultaneous changes in renal function, sodium, potassium values and their interdependence are key parameters in addition to volemia for the assessment of cardiorenal balance. In our paper, an analysis of volemia, electrolytes, and renal function in heart failure was performed using an algorithm based on the ANFIS (Adoptive Neural Fuzzy Inference System), an intelligent approach to renal and heart function monitoring. The study included 90 subjects who were divided into two groups: clinical $(n-80)$ and control $(\mathrm{n}-10)$. The base is composed of parameters B-type natriuretic peptide (NT-proBNP), sodium (Na), potassium (K), ejection fraction (EF), EPI creatinine-cystatin $C$ formula and ANFIS expert system combined in neural network and fuzzy logic network. The results showed that the overall trend of data verification in the network with NT-proBNP, Na and K that we formed is approximately $15 \%$, with which subjects can be classified according to the severity of hypervolemia, electrolyte disturbance and renal function. NT-proBNP ( $\mathrm{pg} / \mathrm{mL}$ ) had the most influence on the EPI creatinine-cystatin C formula. Serum sodium $(\mathrm{Na})$ has the most influence on the ejection fraction (EF).

\section{Introduction}

Renal dysfunction is a common finding in patients with primary and secondary heart disease, and the most common reason for repeated hospitalizations is cardiac decompensation and hypervolemia. It is also known that the therapy used to correct congestion and to improve the pumping function of the heart also affects kidney function [1, 2]. Therefore, an approach for careful monitoring of renal function and electrolyte levels in addition to assessing volemia status has been included in the guidelines for good clinical practice for the treatment of patients with heart failure. However, the therapy suggested in guidebooks is often underdosed or underused due to side effects. The most common side effects of drugs used in the treatment of cardiac decompensation are renal dysfunction and electrolyte disturbance $[3,4,5]$.

The basic parameters for monitoring patients with heart failure are markers of renal function and markers of water-electrolyte balance [6, 7].

Sodium ( $\mathrm{Na}+)$, as an extracellular electrolyte and osmotically active molecule, plays an important role in regulating the water balance. Disorders in serum sodium values are common and are an independent predictor of recurrent hospitalizations due to cardiac decompensation and death after discharge from hospital treatment [8].

Potassium $(\mathrm{K}+)$ is an intracellular cation whose role is reflected in the electrical stimulation of muscle and nerve cells. For cells to function normally, it is necessary that there is a difference between extracellular and intracellular potassium levels. Disorders of serum potassium are common in patients with heart failure. In patients with normal GFR values, serum potassium disturbances occur as part of renin angiotenin aldosterone axis disorder. The disorder is reflected in an imbalance between the sensitivity of 
tubular cells to aldosterone and the activation of the neurohumoral axis. High mortality has been reported in patients with heart failure who have lower serum potassium values than in those with high serum potassium values $[9,10,11]$.

Assessment of renal function is very important for the assessment of outcomes in patients with primary and secondary heart disease and numerous comorbidities. Deterioration of renal function (worsering renal function-WRF) is associated with frequent repeated hospitalizations, prolonged hospital treatment, and high mortality [12].

In clinical practice, serum creatinine is used daily as a marker to assess the strength of glomerular filtration using various formulas. Creatinine is fully filtered in the glomeruli and minimally secreted in the proximal tubules. For this reason, for now, in practice, creatine is the best marker of glomerular filtration, with relatively constant plasma concentrations. It does not show reliability as a marker of the early stages of acute kidney damage because it significantly depends on the volume state and the intensity of catabolic processes. Glomerular filtration is also assessed using cystatin $\mathrm{C}$ in the EPI creatinine-cystatine C formula (Chronic Kidney Disease Epidemiology Collaboration). Cystatin C (CyC) is a marker of not onlyfunctional but also structural damage to the kidneys. Cystatin $\mathrm{C}$ in patients with essential hypertension can be a marker of subclinical, functional and structural damage of the heart, as well as a marker of early renal vascular damage. Therefore, cystatin $\mathrm{C}$ may be a marker of a subclinical phase of cardiorenal disease $[13,14]$.

Hypervolemia is usually manifested by the appearance of peripheral edema, accumulation of fluid in the abdomen and an increase in intra-abdominal pressure after an increase in pressure in the right atrium and a decrease in the functional reserve of the glomeruli. The consequent decrease in the functional reserve of the glomerulus occurs due to the activation of the atrial renal reflex during the increase in circulatory volume and the increased filling pressure of the atria. In chronic conditions of hypervolemia, the natriuresis control mechanism regulated by atrial natriuretic peptide and arginine vasoperesin is ineffective and leads to a paradoxical reduction in diuresis. In this case, the reduced intensity of glomerular filtration and diuresis is a consequence of reduced blood flow through the kidney, which occurs due to vasoconstriction of the afferent arteriole after increased sodium absorption in the proximal tubules $[15,16]$.

Natriuretic peptides are biomarkers that have been suggested by guidebooks to aid in the noninvasive diagnosis of hypervolemia and heart failure. The determination of the B-type natriuretic peptide (BNP) concentration and its precursor have the greatest significance in the diagnosis of heart failure and are independent predictors of mortality in these patients. Chronic heart failure involves resistance to released NT-proBNP, as well as deficits in the active form of BNP. NT-proBNP is also elevated in patients who develop acute kidney injury (AKI) due to acute heart failure, since the end-diastolic stretching of cardiomyocytes leads to its production. Elevated levels of NT-proBNP are commonly found in patients with heart failure and reduced glomerular filtration $[17,18]$. 
The aim of our paper was to analyze volemia, electrolytes, and renal function in heart failure, using an algorithm based on the ANFIS (Adoptive Neural Fuzzy Inference System), an intelligent approach to renal and heart function monitoring.

\section{Methodology}

\subsection{Measuring data}

The study group included 90 subjects older than 18 years of both sexes. Of the total number of analyzed respondents, 10 were healthy respondents of both sexes. The clinical group of remaining n-80 subjects with heart and kidney damage had n-52 men (57.77\%), and n-38 women (42.22\%).

The study was conducted at the Department of Nephrology of the Clinical Center in Niš, Department of Cardiology of Universuty Clinical Center in Niš, Institute of Biochemistry of the Faculty of Medicine in Niš and the Center for Medical Biochemistry of the University Clinical Center in Niš over a 9 month period. Our study protocol was approved by the Ethic Committee of The Faculty of Medicine Nis, University of Nis ( approval number 01-6481-9). All patients have signed the written informed consent. The study has been performed in accordance with the Decleration of Helsinki.

This was a prospective cross-sectional study comparing subjects with associated renal and heart failure or with the existence of a "de novo" or previously diagnosed, clinically manifested cardiovascular disease and with the existence of acute kidney injury or the presence of chronic kidney disease at different stages of evolution. All patients who had malignant disease of any etiology, acute and chronic inflammatory diseases of other organ systems and clinical manifestations of thyroid disease were excluded from the study. Blood samples for routine hematological analysis and biochemical analysis after centrifugation for 15 minutes at $1000 \mathrm{rpm}$ and $5 \mathrm{ml}$ of serum were analyzed by a standard method with commercially available tests. $\mathrm{Na}+, \mathrm{K}+$ electrolyte values were measured on a Roche $9181 \AA$ analyzer with reference values for $\mathrm{Na}+135-150 \mathrm{mmol} / \mathrm{L}$, and for $\mathrm{K}+3.5-5.5 \mathrm{mmol} / \mathrm{L}$. Plasma BNP concentration was determined by enzymatic immunoassay quantitative chemiluminescent microparticle immunoassay CMIA technology on an Abbott Laboratories ${ }^{\circledR}$ apparatus. Antiserum-NT-proBNP micropatriculas were added to the plasma sample, and the reaction was determined as the ratio of the amount of NT-proBNP to the relative light units of RLUs "relative light units". NT-proBNP concentration is expressed in $\mathrm{pg} / \mathrm{ml}$. The limit value for NT-proBNP is $300 \mathrm{pg} / \mathrm{ml}$ was used as a reference in patients with glomerular filtration rate less than $15 \mathrm{ml} / \mathrm{min} / 1.73 \mathrm{~m}^{2}$ calculated using CKD-EPI cystatin C formula. A reference NT-proBNP cutoff value of less than $100 \mathrm{pg} / \mathrm{m}$ was used in patients with glomerular filtration rate if EPIcistC $>90 \mathrm{ml} / \mathrm{min} / 1.73$ $\mathrm{m} 2$. Serum cystatin $\mathrm{C}(\mathrm{Cys} \mathrm{C})$ was determined in plasma using a commercial ELISA kit. Determination of serum cystatin C-based JGF was performed using a reference formula using a calculator [19].

Echocardiographic examinations were performed using a Toshiba Powervision 6000 Tochiba Co® device with a multifrequency phase array transducer $2.0-4.5 \mathrm{MHz}$ transthoracic approach in compliance with all recommendations of good clinical practice [20]. This review determined $\mathrm{EF} \%$ as a functional parameter 
using the Teicholz formula in $\mathrm{M}$ mode or Simpson's rule in volumetric calculation where normal EF values are greater than $50 \%$, cutoff normal values between $40 \%$ and $49 \%$, and low values less than $40 \%$ [21].

\subsection{Neuro-fuzzy method}

In the previous section, we found that although it was a small group of patients, there was a significant correlation between serum electrolytes $(\mathrm{Na}+, \mathrm{K}+)$ and $\mathrm{BNP}$ and cardiac and renal function as assessed by EF (ejection fraction) and CKD-EPIcistC equations for GFR (glomerular filtration rate). We ask the question of what impact the occurrence of imbalance of these parameters has on further monitoring or hospitalization of the patient $[22,23,24]$. To analyze the given problem, we used the adaptive neuro-fuzzy interference system (ANFIS) network type, which is supervised learning with fuzzy logic that is similar to Takagi and Sugeno's approach. The process of learning a neural network with phase logic, Figure 1, represents a complex structural learning of linking input parameters that do not have clearly defined boundaries and their impact with a certain degree of state severity in linking to target values as output parameters $[25,26]$.

\section{Model Description}

We used the structure of the ANFIS network, which we based on the connection of input parameters: BNP $(\mathrm{pg} / \mathrm{mL}), \mathrm{Na}+(\mathrm{mmol} / \mathrm{L})$ and $\mathrm{K}+(\mathrm{mmol} / \mathrm{L})$ with one output parameter $\mathrm{EF}(\%)$ or EPIcistC $(\mathrm{ml} / \mathrm{min} / 1.73 \mathrm{~m} 2)$ in beck propagation (BP), Figure 1. By normalizing with the min-max method $[27,28$, 29], we adjusted the values of all parameters (input and output) to the range of values of the base parameters [0,1], to ensure the formation of a network of maximum precision and reliability and thus removed the possibility of dominance of individual data due to approximation and neglect of data values due to different orders of magnitude. Patient data were classified into three groups of ANFIS database data. These are: training data, testing data and checking data. The structure of the ANFIS network determines the manner and time of training. The network contains five hidden layers with different numbers of neurons [30,31,32]. The neurons in the layers are related by weighting factors $\omega_{i}, i=1, \ldots 27$, which change and adjust during training in the back propagation standard mean square error (MSE).

The input layer data (NT-proBNP, $\mathrm{Na}+$ and $\mathrm{K}+$ ) are adapted due to their range of optimal values by distributing the trapezoidal membership function to the values of the neurons of the next layer of the neural network. The influence of the trapezoidal membership function is such that the value of the input parameter stratifies into three different areas. The NT-proBNP parameter on the

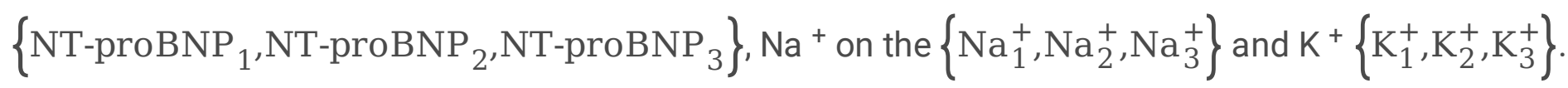
The stratified values of individual parameters NT-proBNP, $\mathrm{Na}^{+}$and $\mathrm{K}^{+}$are assigned to the phase of the rule (fuzzy rule) of the form: 


\section{if (NT-proBNP is NT-proBNP $)$ and $\left(\mathrm{Na}^{+}\right.$is $\left.\mathrm{Na}_{j}^{+}\right)$and $\left(\mathrm{K}^{+}\right.$is $\left.\mathrm{K}_{k}^{+}\right)$then $V_{l}=$ $\mathrm{c}_{1} \cdot \mathrm{NT}-\operatorname{proBNP}_{i}+\mathrm{c}_{2} \cdot \mathrm{Na}_{j}^{+}+\mathrm{c}_{3} \cdot \mathrm{K}_{k}^{+}, \quad i, j$ or $k=\{1,2,3\}$ and $l=27$.}

The third layer normalizes the input value of a single neuron of the third layer with the sum of all values of neurons of the third layer $\omega_{l}=\omega_{l} / \sum_{m=1}^{27} \omega_{m=1}, l=1, \ldots 27$. The values normalized in this way are defined by the output membership function (Outputmf), the sum of which determines the value of the output $O_{l}=\otimes_{l} V_{l}, l=1, \ldots 27$. In the fifth layer, the final value of one output parameter is determined during training as the sum of the values of the fourth layer, $\sum_{l=1}^{27} O_{l}=\sum_{l=1}^{27} \bigotimes_{l} V_{l}$.

The selection of the output parameter is reduced to one and represents either the EPIcisC or EF parameter. The structure of ANFIS requires that the training time of the network is realistic during training in 1000 epochs with a tolerance error for a mean square error (MSE) of 0.0005. This specifically selected structure with the parameters NT-proBNP, $\mathrm{Na}^{+}$and $\mathrm{K}^{+}$leads to the accuracy of the formed network during training, checking on test data (testing data, checking data) and checking is quite consistent and ranges in accuracy values of approximately $15 \%[33,34]$.

\section{Results}

\section{Implementing the model}

The learning algorithm of ANFIS leads to the formation of a model by connecting the given input and output parameters of the respondents. The ANFIS system formed in this way encourages the use of neural networks in the earlier stages of disruption of individual parameters and indicates the need for faster clinical processing of individual subjects. Figure 2 indicates the dependence of one output parameter as a function of two input parameters. The formed three-dimensional surfaces indicate the socalled neuro fuzzy mapping that confirms the following regularities. The area between the green lines indicates the value of $\mathrm{K}+$ clinically stable subjects with certain normalized values of the parameter $\mathrm{K}+$ in the range from 0.20 to 0.60 (area between the green lines), Figure 2a), d) and f). Values of $\mathrm{Na}+$ in the range of 0.44 to 1.00 (area between blue lines), Figure 2b), c) and e), and NT-proBNP in the range of 0.30 (yellow line) to 0.60 (red line), Figure 2. a), b), c) and e) EF parameter values below 0.5 (orange horizontal line) indicate patients with a serious adverse event, while EF values above 0.5 indicate patients who are at risk of an adverse event, Figure 2. a), b) and c). Values for EPIcistC below 0.58 (pink) indicate renal failure of varying degrees, Figure $2 d$ ), e) and f). The dominance of some colors shows that patients with parameters that cause the appearance of yellow colors have heart failure with preserved ejection fraction (HFpEF), while patients who have parameters on horizontal axes that lead to blue colors have heart failure with reduced ejection fraction (HfrEF) and require greater supervision and hospitalization, (Figure 2) $[35,36,37,38]$. 


\section{Characteristics of respodnets}

In this study, an ANFIS model based on a neural network with fuzzy logic was applied to predict renal function and hydroelectrolyte disturbance in patients with heart damage. The usual statistical methods did not find a statistically significant difference in age between healthy subjects who had an average age of $69.55 \pm 32.01$ years and subjects with heart and kidney damage who had an average age of $70.72 \pm$ 9.26 years $(p=0.286)$. No statistically significant difference was found in the values of electrolyte status parameters shown in Table 1 in subjects with heart and kidney damage and in healthy subjects. A statistically significant increase in NT-proBNP $(p<0.001)$ and cystatin $C(p<0.001)$ values was found between healthy subjects and subjects with heart and kidney damage (Mann-Whitney $U$ test).

Table 1

Demographic and laboratory characteristics of respondents

\begin{tabular}{|llllll|}
\hline Parameters & $\mathrm{Na}(\mathrm{mmol} / \mathrm{L})$ & $\mathrm{K}(\mathrm{mmol} / \mathrm{L})$ & $\mathrm{NT}-\mathrm{pro} \mathrm{BNP}(\mathrm{pg} / \mathrm{mL})$ & CistatinC(mg/L) & Age(years) \\
\hline Min. value & 123 & 2.4 & 10 & 1.73 & 18 \\
\hline Max. value & 150 & 7.8 & 5000 & 0.21 & 88 \\
\hline Mean & 137.90 & 4.84 & 1275.77 & 3.33 & 65.98 \\
\hline SD & 4.57 & 0.97 & 1533.89 & 0.825 & 15.74 \\
\hline
\end{tabular}

Table 2 shows the parameters of heart and kidney function in all subjects. A statistically significant difference was found in the values of EF $(p<0.001)$ and Epi formula $(p<0.001)$ by analysis of healthy and diseased subjects (Mann-Whitney U test).

Table 2

Glomerular filtration and functional status of the subjects' hearts

\begin{tabular}{|lllll|}
\hline Parameters & Min. value & Max. value & Mean & SD \\
\hline EF \% & 12 & 75 & 72.8 & 15.04 \\
EPI cistatin C & 14 & 146 & 50.20 & 37.88 \\
$\left(\mathrm{ml} / \mathrm{min} / 1.73 \mathrm{~m}^{2}\right)$ & & & & \\
\hline
\end{tabular}

\section{Discussion}

To obtain more accurate results, we used Al machine learning to classify the collected data. Cardiorenal syndrome is a complex syndrome characterized by salt and water retention and activation of various neurohumoral mechanisms. In fact, the kidney and the heart are interconnected by regulatory mechanisms that are important for maintaining homeostasis in the body [39]. Disorder in the function of 
these mechanisms is an introduction to the vicious circle of causes and consequences, which is characterized by a higher probability of premature death and deterioration of kidney and heart function [40]. Since this outcome is more common in cardiorenal syndrome than if there is isolated heart and kidney damage, it is important to identify high risk patients as early as possible to apply preventive and therapeutic measures [41].

Type $B$ natriuretic peptide (BNP) is a marker of neurohumoral stimulation whose activity is associated with inhibition of sympathetic nerve activity and the renin angiotensin system axis. NT-proBNP in healthy individuals, even in the case of dietary salt intake, has a protective role for kidney and heart function, while in the early stages of heart and kidney disease, it induces natriuresis and diuresis, and in advanced stages of the disease, this neurohormone becomes ineffective in regulating hypervolemia. The explanation lies in the fact that at the renal level, NT-proBNP at physiological concentrations acts by increasing the strength of glomerular filtration and directly inhibits the tubuloglomerular feedback response, which first inhibits sodium resorption at the distal tubule and then at the proximal tubule, reduces intrarenal vascular resistance but has no effect on the permeability of intrarenal blood vessels [42]. The consequence of the physiological action of the NT-proBNPa molecule is an increase in the volume of excreted urine and an increase in sodium excretion without affecting blood pressure and heart rate [43]. In addition, NT-proBNP plays an important role in the prevention of chronic renal impairment in patients with asymptomatic chronic heart failure due to its effect on intrarenal blood flow. The paradoxical role of NT-proBNP in patients with heart failure by decreased diuresis, natriuresis, and increased vasoconstriction leads to deterioration of heart and kidney function and the general condition of the patient despite a significantly high concentration of the biologically inactive form of circulating BNP [44]. In addition to the fact that the clearance of NT-pro BNP depends on several mechanisms that have not been fully elucidated, it is certain that this protective counterregulatory neurohumoral mechanism is ineffective in patients with heart and kidney damage [45]. The consequences are salt and water retention, hypertension, concentric left ventricular hypertrophy and heart fibrosis.

In our study, NT-proBNP was a useful biomarker for assessing the progression of cardiac and renal dysfunction in our subjects with cardiorenal syndrome. The results of our study showed that the overall trend of data verification in the network with NT-proBNP, Na and $\mathrm{K}$ that we formed is approximately $15 \%$, with which subjects can be classified according to the severity of hypervolemia, electrolyte disturbance and renal function. Electrolyte disturbance is a common finding in patients with heart failure and a consequence of the use of diuretics and disorders of neurohumoral activation or a combination of these factors. Hyponatremia is common in patients with acute cardiac decompensation due to dilution and impaired excretion of free water or as a consequence of sodium depletion. Hyperkalemia is often the result of the use of RAAS blockers, mineralocroticode receptor antagonists, or potassium-sparing diuretics. Hypokalemia is also a common finding and is a consequence of magnesium deficiency and the use of Henle loop diuretics [46]. However, in addition to hypokalemia, Henle's loop diuretics can lead to hypovolemia and deterioration of renal function, which requires a reduction in the administered dose of diuretics, which is the basic and first drug in people with acute cardiac decompensation [47]. There is no standardized method in clinical practice that would prove the degree of decongesting during 
hospitalization, and often due to the lack of appropriate criteria for defining adequate decongesting, patients require frequent check-ups in an outpatient setting.

Assessing the vital risk of patients and recurrence of decompensation of patients with combined heart and kidney damage involves extensive and repeated diagnosis, many wandering in terms of determining the causes and consequences and further treatment planning even by very experienced doctors. This model may be superior to the traditional diagnostic approach due to its contribution to more accurate and rapid diagnostic interpretation and better planning of further patient treatment.

The way in which high values of EPIcistC and EF indicate the risk of adverse events is shown in Figure 2. $1-f$. Dependence on the parameters of NT-proBNP, Na+ and K+ patients based on ANFIS results. It has been shown that both low values of $\mathrm{Na}+$ and $\mathrm{K}+$ lead to worsening of the condition and vital endangerment of patients.

Our work aims to fill a gap in specific systematized predictive tools in high-risk patients with associated heart and kidney damage. After rigorous validation, this tool will help to predict serious adverse events before they occur and thus improve the treatment outcome of these patients. The predictions obtained from this model can help optimize preventive strategies and intensive monitoring for patients identified as at risk for electrolyte disturbance and hypervolemia. To identify the risk of occurrence, the model identifies a prognostic biomarker by random regression from the total data set.

\section{Conclusion}

Serum potassium disturbances are associated with advanced heart failure and reduced prognosis. Cardiorenal syndrome is used for the estimation of heart failure and kidney disease. There are numerous factors that contribute to the maintenance of disturbed values of potassium in cardiorenal syndrome. Definitely, it is independent of many influences, and the balance of serum potassium is more important than sodium in cardiorenal syndrome. In this study, the potassium balance in cardiorenal syndrome was analyzed by the adaptive neuro-fuzzy inference system or ANFIS. ANFIS is suitable for nonlinear systems with highly redundant data. Although there are encouraging advances around this unsolved clinical problem, further investigation should consider the progressive inclusion of patients with advanced renal impairment to allow a better understanding of cardiorenal syndrome.

\section{Study Limitation}

This method dealt with the prediction of "incidents" on a small number of heterogeneous high-risk subjects. Future research should explore the potential for a long-term risk solution.

\section{Declarations}

\section{Author contributions}


D.T. participated in the design of the study and drafted and reviewed the manuscript. K.Đ. end DF participated in its design and coordination performed the statistical analysis. S.G., Z. D, end S.R. participated in the design of the study and acquisition of data. All authors read and approved the final manuscript.

\section{Competing interests}

The authors declare no competing interests.

\section{Additional information}

Correspondence and requests for materials should be addressed to D.T.

Reprints and permissions information is available at www. Nature.com/reprints.

\section{Publisher's note}

Springer nature reains neutral with regard to jurisdictional claims in published maps and institutional affilations.

\section{References}

1. Ronco, C., Cicoira, M., \& Mc Cullough, P. A. Cardiorenal syndrome type 1: pathophysiological crosstalk leading to combined heart and kidney dysfunction in the setting of acutely decompensated heart failure. J Am Coll Cardiol. 60 (12), 1031-104 (2012). https://doi.org/10.1016/j.jacc.2012.01.077

2. Zannad,F.\& Rossignol, P. Cardiorenal syndrome revisited. Circulation.138:929-944(2018). https://doi.org/10.1161/CIRCULATIONAHA.117.028814

3. Ronco, C. et al for the Acute Dialysis Quality Initiative (ADQI) consensus group. Cardio-renal syndromes:report from the consensus conference of the Acute Dialysis Quality Initiative.Eur Heart J.31,703-711 (2010).https://doi.org/10.1093/eurheartj/ehp507

4. Agewall, S. Adherence to guidelines and regisry data. Eur Heart J Cardiovascular Pharmacother.3,183-184(2017). https://doi.org/10.1093/ehjcvp/pvx027

5. Ambrosy, A.P. \& Gheorghiade, M. Real-world dosing of evidence-based medications for heart failure: embracing guideline recommendations and clinical judgment. Eur J Heart Fail.19,1424-1426 (2017). https://doi.org/10.1002/ejhf.915

6. Tate, S., Griem, A., Durbin-Johnson, B., Watt, C.\& Schaefer, S. Marked elevation of B-type natriuretic peptide in patients with heart failure and preserved ejection fraction. J Biomed Res. 28(4), 255-261 (2014). https://doi.org/10.7555/JBR2820140021. 
7. Pan, A. The real-world evidence of heart failure comorbidities. Eur J Heart Fail.19(3),434. (2017). https://doi.org/10.1002/ejhf.751

8. Dunalp, M.E. et al.Current management of Hyponatremia in Acute heart Failure: A report from the Hyponatremia registry for patients With Euvolemic and Hypovolemic Hyponatremia. J AmHeart Assoc.6(8) (2017). https://doi.org/10.1161/JHA.116.005261

9. Polson, M. et al. Clinical and Economic Impact of Hyperkaliemia in Patients with Chronic Kidney Disease and Heart Failure. J Manag Car Spec Pharm. 23(4 Suppl):S2-S9 (2017). https://doi.org/10.18553/jmcp.2017.23.4-a.s2a.

10. Tromp, J. et al. Serum Potassium Levels and Outcome in Acute Heart Failure (Data fro the PROTECT and COACH Trials). Am J Cardiol. 119 (2), 290-296 (2017).

https://doi.org/10.1016/j.amjcard.2016.09.038.

11. Rossignol, P. et al. Emergency management of severe hyperkalemia: Guideline for best practice and opportunities for the future. Pharmacol Res. 113, 585-591 (2016).

https://doi.org/10.1016/j.phrs.2016.09.039.

12. Kervella, D. et al. Cystatin C Versus.Creatinine for GFR Estimation in CKD Due to Heart Failure. Am J Kidney Dis. 69(2), 321-323 (2017). https://doi.org/10.1053/j.ajkd.2016.09.016.

13. Stevens, L.A.\& Levey, A.S. Measured GFR as a confirmatory test for estimated GFR. J Am Soc nephrol.20:2305-2313 (2009). https://doi.org/10.1681/ASN.2009020171

14. Dupont, M., Wu, Y., Hazen, L.S. \& Tang, W.H.W. Cystatin C Identifies Patients With Stable Chronic Heart Failure at Increased Risk for Adverse Cardiovascular Events. Circ Heart Fail. 5,602-609 (2012). https://doi.org/10.1161/CIRCHEARTFAILURE.112.966960

15. Martens, P. \& Mullens, W. Spot Urinary Sodium in Decompensated Heart Failure as a Prognostic Metric for Successful Ambulatory Decongestion. J Card Fail.24:355-356 (2018). https://doi.org/10.1016/j.cardfail.2018.04.002

16. Chioncel, O., et al.,on behalf on the ESC-EORP HFA Heart Failure Long-term Registry Investigators. Acute heart failure congestion and perfusion status-impact of the clinical classification on inhospital and long-term outcomes;insights from the ESC-EORP HFA Heart Failure Long-Term Registry. EurJHeartFaikbi>.</bi>21(11):1338-1352(2019). https://doi.org/10.1002/ejhf.1492.

17. Horii, M. et al. Prognostic value of B-type natriuretic peptide and its amino-terminal proBNP fragment for cardiovascular events with stratification by renal function. J Cradiol.61:410-416(2013). https://doi.org/10.1016/j.jjcc.2013.01.015

18. Takase, H. et al. B-type natriuretic-peptide levels and cariovascular risk in patients with diastolic dysfunction on chronic hemodialysis:cross-sectional and observational studies. Nephrol Dial Transpant.26:683-690(2011). https://doi.org/10.1093/ndt/gfq408.

19. http://www.kidney.org/professionals/KDOQI/gfr_calculator

20. Boyer, N.M. et al. Trends in Clinical, demographic, and Biochemical Characteristics of Patients With Acute Myocardial infarction From 2003 to 2008: A repost from the American heart Association get 
With The Guidelines Coronary Artery Disease Program. J Am Heart Assoc.1(4):e001206.(2012). https://doi.org/10.1161/JAHA.112.001206.

21. Wandt, B., Bojo, L., Tolagen, K. \& Wranne, B. Echocardiographic assessment of ejection fraction in left ventricular hypertrophy.Heart.82(2):192-198(1999). https://doi.org/10.1136/hrt.82.2.192

22. Shahjalal, M., Mitra, D.J., Sulta, A., Mitra, N.K. \& Khodadad Khan, A.F.M. Measuring Risk of Diabetic: A Fuzzy Logic Approach, Progress in Nonlinear Dynamics and Chaos. 4 (1), 23-33 ISSN: 2321-9238 (2016).

23. Katigari, M.R., Ayatollahi, H., Malek, M. \&Haghighi, M.K. Fuzzy expert system for diagnosing diabetic neuropathy, World J Diabetes. 8(2): 80-88 (2017). https://doi.org/10.4239/wjd.v8.i2.80

24. Vosoulipour, A., Teshnehlab, M.\&Moghadam, H. A. Classification on Diabetes Mellitus Data-set Based-on Artificial Neural Networks and ANFIS. 4th Kuala Lumpur International Conference on Biomedical Engineering. 27-30 (2008). https://doi.org/10.1007/978-3-540-69139-6_12

25. Bressan, G.M., Flamia de Azevedo, B.C.\&Molina de Souza, R. A. Fuzzy Approach for Diabetes Mellitus Type 2 Classification. Braz. Arch. Biol. Technol. 63(2020). e20180742, http://dx.doi.org/10.1590/1678-4324-2020180742 ISSN 1678-4324

26. Jang, J.S. ANFIS: adaptive-network-based fuzzy inference system. IEEE transactions on systems, man, and cybernetics.23(3):665-85(1993). https://doi.org/10.1109/21.256541

27. Furundzic, D., Djordjevic, M., \& Bekic, AJ. Neural networks approach to early breast cancer detection. J Syst Architect. 44 (8): 617-633(1998). https://doi.org/10.1016/S1383-7621(97)00067-2

28. Furundzic, D., Stankovic, S., Jovicic, S., Punisic, S \& Subotic, M. Distance based resampling of imbalanced classes: With an application example of speech quality assessment, Eng. Appl. Artif. Intell, 64, 440-461(2017). https://doi.org/10.1016/j.engappai.2017.07.001

29. Übeyli E.D., Automatic diagnosis of diabetes using adaptive neuro-fuzzy inference systems, Expert Syst. J. Knowl. Eng. 27(4), 259-266 (2010). https://doi.org/10.1111/j.1468-0394.2010.00527.x

30. Priyadarshini, L. \& Shrinivasan, L. Design of an ANFIS based Decision Support System for Diabetes Diagnosis. <bi>International Conference on Communication and Signal Processing (ICCSP) </bi> (2020). https://doi.org/10.1109/iccsp48568.2020.91821

31. Alby, S.B.L. \& Shivakumar, B.L.Prediction of Type 2 Diabetes using Optimized ANFIS with Genetic Algorithm and Particle Swarm Optimization, (IJCSIT) International Journal of Computer Science and Information Technologies, Vol. 7 (4) 2090-2095 (2016) ISSN:0975-9646

32. Abushariah, M.A.M., Alqudah, A.A.M., Adwan, O.Y\&Yousef, R.M.M. Automatic Heart Disease Diagnosis System Based on Artificial Neural Network (ANN) and Adaptive Neuro-Fuzzy Inference Systems (ANFIS) Approaches. Journal of Software Engineering and Applications, 7(12), (2014).https://doi.org/10.4236/jsea.2014.712093

33. Ghosh, G., Sandipan, R. \& Merdji., A. A proposed health monitoring system using fuzzy inference system. Proc I Mech E Part H.J Engineering in Medicine.1-8 (2020). https://doi.org/10.1177/0954411920908018 
34. Dogantekin, E., Dogantekin, A., Avci, D., \& Avci, L. An intelligent diagnosis system for diabetes on Linear Discriminant Analysis and Adaptive Network Based Fuzzy Inference System: LDA-ANFIS. Digital Signal Processing.20(4),1248-1255, (2010). https://doi.org/10.1016/j.dsp.2009.10.021

35. Samira, K.\&Ahmad, J. A New Artificial Intelligence Method for Prediction of Diabetes Type 2. Bulletin de la Société Royale des Sciences de Liège. 85.376 - 391(2016). https://doi.org/10.25518/00379565.5420

36. Yadollahpour, A., Nourozi, J., Mirbagheri, S.A., Simancas-Acevedo, E.\&Trejo-Macotela, F.R.Designing and Implementing an ANFIS Based Medical Decision Support System to Predict Chronic Kidney Disease Progression. Front Physiol. 9, 1753 (2018). https://doi.org/ 0.3389/fphys.2018.01753.

37. Naumović, R., Furundzić, D., Jovanović, D., Stosović, M., Basta-Jovanović, G. \& Lezaić, V.Application of artificial neural networks in estimating predictive factors and therapeutic efficacy in idiopathic membranous nephropathy. Biomedicine \&Pharmacotherapy.64,633-638 (2010). https://doi.org/10.1016/j.biopha.2010.06.003

38. Ogrizović-Simić, S., Furundzić, D., Lezaić, V., Radivojević, D., Blagojević, R.\& Djukanović, Lj. Using ANN in Selection of the Most Important Variables in Prediction of Chronic Renal Allograft Rejection Progression. Transplant Proc.31(1-2), 368 (1999). https://doi.org/10.1016/s0041-1345(98)01665-0

39. Gnanaraj, J.F., Haehling von, S., Anker, D.S., Raj, S.D. \&Radhakrishnan, J. The relevance of congestion in the cardiorenal syndrome. Kidney Int.83,384-391(2013). https://doi.org/10.1038/ki.2012.406

40. Rubio-Gracia, J., Gimenez-Lopez, I., Sanchez-Marteles, M., Josa-Laorden. C.\& Perez-Calvo, J. Intraabdominal pressure and its relationship with markers of congestion in patiensts admitted for acute decompensated heart failure. Heart vessels.35(11):1545-1556 (2020).

https://doi.org/10.1007/s00380-020-01634-9

41. Lim, S.Y., \& Kim, S. Pathophysiology of Cardiorenal Syndrome and Use of Diuretc and Ultrafiltration as Volume Control. Korean Cic J.51(8):656-667(2021). https://doi.org/10.4070/kcj.2021.0996

42. Okamoto, R., Ali, Y., Hashizume, R.\& Suzuki, N., Ito, M.BNP as a Major Player in the Heart-Kidney Connection. Int J Mol Sci.20(14):3581(2019). https://doi.org/10.3390/ijms20143581.

43. Levin, E.R., Gardner, D.G.\& Samson, W.K. Natriuretic peptides. N Engl J Med. 339(5):321-328 (1998). https://doi.org/10.1056/NEJM199807303390507

44. Pastural-Thaunat, M. et al. Relative Change in NT-proBNP Level: An Important Risk predictor of Cardiovascular Congestion in Hemodialysis patients. Nephron Extra.2 (1),311-318(2012). https://doi.org/10.1159/000343897.

45. Parcha, V. et al. Chronobiology of Natriuretic Peptides and Blood Pressure in Lean and Obese Individuals. J Am Coll Cardiol.77(18),2291-2303(2021). https://doi.org/10.1016/j.jacc.2021.03.291.

46. Rossignol, P., Coats, A.J., Chioncel, O., Spoetini, I.\& Rosano, G. Renal function, electrolytes and congestion monitoring in heart failure. Eur Heart J Suppl.21,M25-M31(2019). https://doi.org/10.1093/eurheartj/suz220.

47. Husan-Syed, F.et al. Congestive nephropathy: a neglected entity? proposal for diagnostic criteria and future persectives. ESC Heart Fail.8(1):183-203(2021). https://doi.org/10.1002/ehf2.13118. 


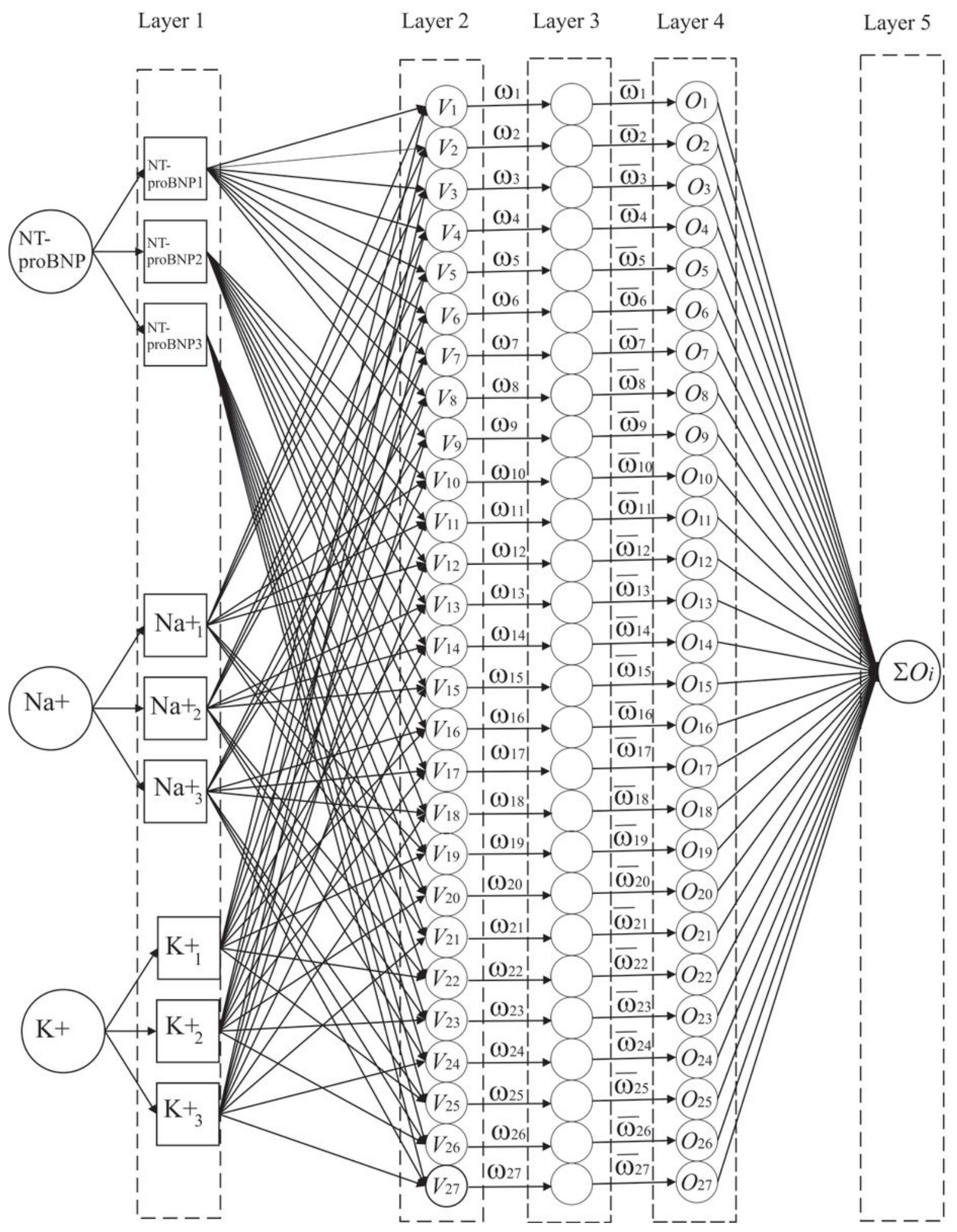

Figure 1

Representation of the ANFIS network used for training on BPU, $\mathrm{Na}+\mathrm{K}+$ parameters with the aim of obtaining EF (\%) or EPI cystatin $\mathrm{C}$ as control parameters of cardiac and renal function. 

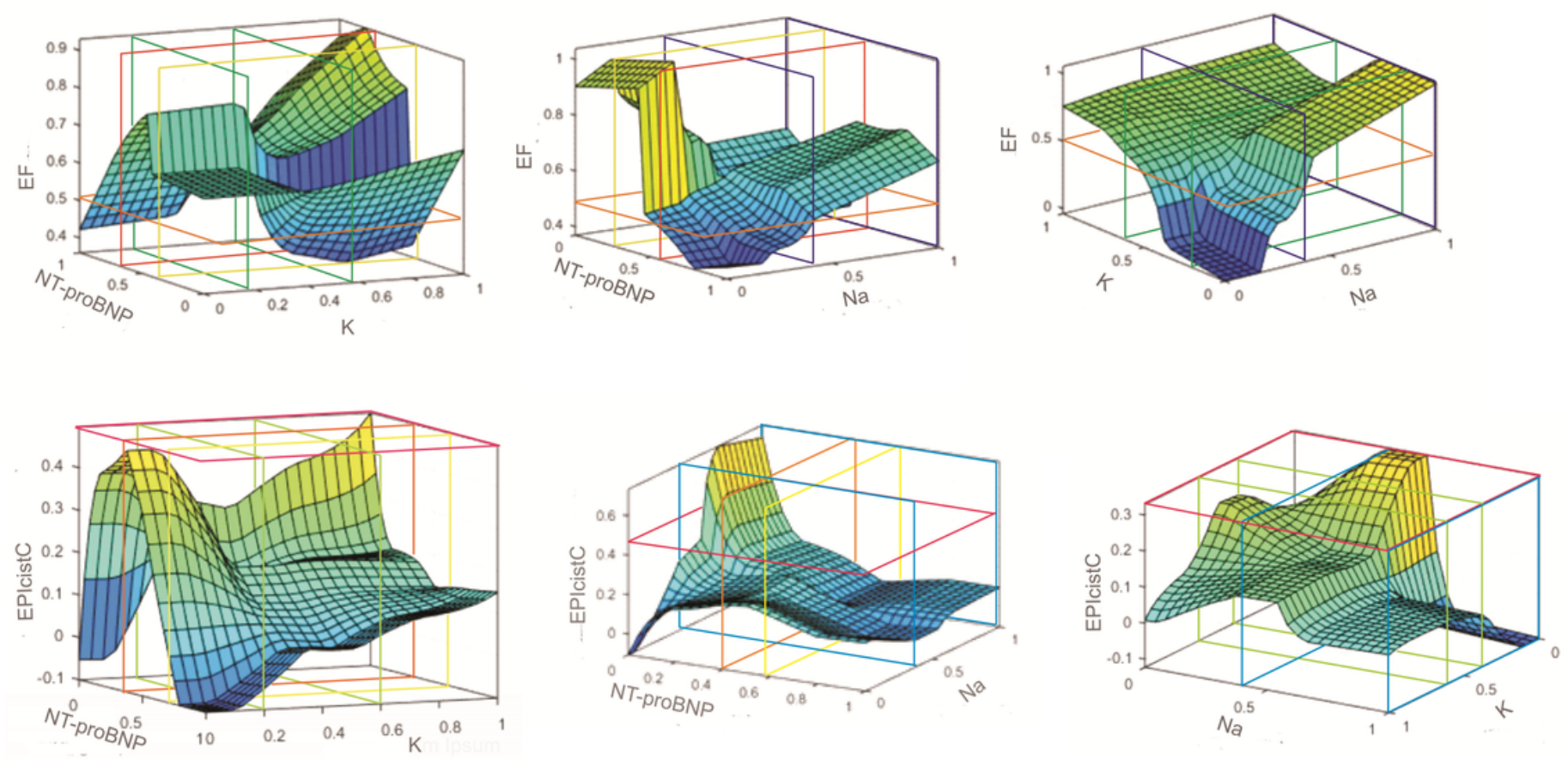

Figure 2

Estimation of ANFIS network of interdependence areas of parameter values: EF values as a functional dependence a) NT-pro BNP and $\mathrm{K}^{+}$, b) BNP and $\mathrm{Na}^{+}$ic) $\mathrm{Na}^{+}$and $\mathrm{K}^{+}$, and EPIcistC as a functional dependence d) NT-proBNP and $\mathrm{K}^{+}$, e) NT-proBNP and $\mathrm{Na}^{+}$f) $\mathrm{Na}^{+}$and $\mathrm{K}^{+}$ 